\title{
Regulation of Sucrose non-Fermenting Related Kinase 1 genes in Arabidopsis thaliana
}

\author{
Sarah P. Williams ${ }^{1}$, Padma Rangarajan ${ }^{1}$, Janet L. Donahue ${ }^{1}$, Jenna E. Hess ${ }^{2}$ and Glenda E. Gillaspy ${ }^{1 *}$ \\ Department of Biochemistry, Virginia Tech, Blacksburg, VA, USA \\ ${ }^{2}$ Alliance Biosciences, Richmond, VA, USA
}

\section{Edited by:}

Astrid Wingler, University College

London, UK

Reviewed by:

Sonia Gazzarrini, University of

Toronto, Canada

Eleazar Martínez, Universidad

Nacional Autónoma de México,

Mexico

*Correspondence:

Glenda E. Gillaspy, Department of

Biochemistry, Virginia Tech,

Blacksburg, VA 24061, USA

e-mail: gillaspy@vt.edu
The Sucrose non-Fermenting Related Kinase 1 (SnRK1) proteins have been linked to regulation of energy and stress signaling in eukaryotes. In plants, there is a small SnRK1 gene family. While the SnRK1.1 gene has been well studied, the role other SnRK1 isoforms play in energy or stress signaling is less well understood. We used promoter:GUS analysis and found SnRK1.1 is broadly expressed, while SnRK1.2 is spatially restricted. SnRK1.2 is expressed most abundantly in hydathodes, at the base of leaf primordia, and in vascular tissues within both shoots and roots. We examined the impact that sugars have on SnRK1 gene expression and found that trehalose induces SnRK1.2 expression. Given that the SnRK1.1 and SnRK1.2 proteins are very similar at the amino acid level, we sought to address whether SnRK1.2 is capable of re-programming growth and development as has been seen previously with SnRK1.1 overexpression. While gain-of-function transgenic plants overexpressing two different isoforms of SnRK1.1 flower late as seen previously in other SnRK1.1 overexpressors, SnRK1.2 overexpressors flower early. In addition, SnRK1.2 overexpressors have increased leaf size and rosette diameter during early development, which is the opposite of SnRK1.1 overexpressors. We also investigated whether SnRK1.2 was localized to similar subcellular compartments as SnRK1.1, and found that both accumulate in the nucleus and cytoplasm in transient expression assays. In addition, we found SnRK1.1 accumulates in small puncta that appear after a mechanical wounding stress. Together, these data suggest key differences in regulation of the SnRK1.1 and SnRK1.2 genes in plants, and highlights differences overexpression of each gene has on the development of Arabidopsis.

Keywords: SnRK1, trehalose, sugar-sensing, energy, gene expression

\section{INTRODUCTION}

The Sucrose non-Fermenting Related Kinase 1 (SnRK1) family of proteins has been linked to regulation of energy metabolism and stress signaling in diverse types of eukaryotes (Halford and Hey, 2009; Coello et al., 2011; Tsai and Gazzarrini, 2014). All plants surveyed contain $S n R K 1$ genes that are structurally and functionally analogous to their yeast and mammalian counterparts, sucrose non-fermenting 1 (SNF1) and AMP-activated protein kinase $(A M P K)$, respectively. SNF1, AMPK, and SnRK1 are Ser/Thr protein kinases that are considered to function as fuel gauge sensors that sense cellular carbohydrate status and/or AMP/ATP levels in order to maintain growth in response to available energy (Halford and Paul, 2003; Rolland et al., 2006; Hey et al., 2010; Ghillebert et al., 2011). SnRK1 proteins carry out their function as part of heterotrimeric protein complexes, binding to $\beta$ and $\gamma$ subunits (Lumbreras et al., 2001; Gissot et al., 2006; Polge et al., 2008) or to a hybrid $\beta \gamma$ subunit in plants (Kleinow et al., 2000; Lopez-Paz et al., 2009; Ramon et al., 2013).

The small Arabidopsis SnRK1 gene family is composed of three genes, SnRK1.1, SnRK1.2, and SnRK1.3 (Baena-Gonzalez et al., 2007). While SnRK1.3 is considered a non-expressed pseudogene, the roles of SnRK1.1 and SnRK1.2 were delineated in seminal work by Baena-Gonzalez using a genetics approach in Arabidopsis thaliana. Overexpression of the SnRK1.1 gene re-programs metabolism such that flowering and senescence of mature plants is delayed (Baena-Gonzalez et al., 2007). The delay in flowering and senescence effectively lengthens the lifespan of the plant, most likely through the combined direct protein phosphorylation of SnRK1 substrates, regulation of transcription in the nucleus (Baena-Gonzalez et al., 2007), and post-transcriptional regulation of target genes (Confraria et al., 2014). Some of the known SnRK1 substrates are key metabolic enzymes such as sucrose phosphate synthase, nitrate reductase, and HMG-CoA reductase (Halford et al., 2003), while others such as FUS3 are transcription factors (Tsai and Gazzarrini, 2012a). FUS3 and SnRK1.1 interact to regulate embryonic-to-vegetative and vegetative-to reproductive phase transitions and lateral organ development (Tsai and Gazzarrini, 2012a). FUS3-overexpression delays vegetative growth and flowering by increasing levels of $\mathrm{ABA}$, while repressing GA biosynthesis and ethylene signaling (Gazzarrini et al., 2004). Therefore, SnRK1 may regulate post-embryonic development through regulation of hormone biosynthesis and signaling. Analysis of SnRK1 genetic mutants in Arabidopsis showed that SnRK1.1 and SnRK1.2 genes have partially redundant functions, 
and when expression of both genes are reduced, seedling growth under low energy conditions is compromised and mature plants undergo growth deprivation, early flowering, and early senescence (Baena-Gonzalez et al., 2007). Comparable phenotypes were also shown in moss (Physcomitrella patens), in which a double mutant lacking the two SnRK1 genes has accelerated development and early senescence (Thelander et al., 2004).

Other gain-of-function studies have shown that SnRK1.1 overexpressors are altered in ABA as well as sugar signaling pathways (Jossier et al., 2009). Recent work has shown that SnRK1.1 and SnRK1.2 interact with two clade A type 2C protein phosphatases, established repressors of the ABA signaling pathway. Inactivation of SnRK1.1 by these phosphatases may allow for the coordinated activation of ABA and energy signaling (Rodrigues et al., 2013). The SnRK1.1 and SnRK1.2 proteins are $81 \%$ identical and contain a similar arrangement of functional domains including an $\mathrm{N}$ terminal kinase domain, a Ubiquitin associated (UBA) domain, and a C-terminal kinase associated domain. The regions outside the kinase domain have been shown to facilitate SnRK1 protein interactions with various proteins, indicating that SnRK1 proteins may be part of several different protein complexes (Singh et al., 2009; Ng et al., 2013; Lin et al., 2014; Mohannath et al., 2014).

Interestingly, biochemical data has indicated that SnRK1 activity in plant cells is mostly a function of the SnRK1.1 gene product (Jossier et al., 2009), thus the SnRK1.2 gene may play a minor or restricted role in regulating most plant metabolism, stress and/or energy sensing. The Arabidopsis SnRK1.2 gene has been overexpressed in $N$. benthamiana, where overexpression leads to enhanced resistance to geminiviral infection, although at the cost of adverse effects on plant growth (Mohannath et al., 2014). Although the network of genes regulated by SnRK1 has been elucidated (Baena-Gonzalez et al., 2007), our understanding of how the $\operatorname{SnRK1.1}$ and $S n R K 1.2$ genes themselves are regulated and function with respect to one another has not been addressed. The $S n R K 1$ genes have been shown to be expressed throughout development, including within the meristem and leaf primordia (Takano et al., 1998; Pien et al., 2001; Bradford et al., 2003; Fragoso et al., 2009; Bitrian et al., 2011), however very little spatial information on the expression of different $S n R K 1$ genes within a species exists.

Given that there are multiple cDNAs of both $S n R K 1$ genes expressed in plants, we sought to address the spatial regulation of $\operatorname{SnRK1.1}$ and SnRK1.2 in Arabidopsis, how different sugars impact expression of these genes, and how different SnRK1 protein isoforms alter plant growth and development when overexpressed. Our data show that $S n R K 1.2$ gene expression is spatially restricted within Arabidopsis, and can be induced by trehalose, but not other sugars. When overexpressed, a SnRK1.2-green fluorescent protein (GFP) gene fusion alters development in a manner opposite in nature to overexpression of $S n R K 1$.1-GFP. These data suggest that $S n R K 1.2$ may have a unique function in plants that warrants further investigation.

\section{MATERIALS AND METHODS PLANT GROWTH CONDITIONS}

Arabidopsis thaliana ecotype Columbia and Landsberg erecta (Ler) plants were used for all experiments. For seedling growth experiments, seeds were surface-sterilized and plated on $0.5 \times$ Murashige and Skoog (MS) salts $+0.8 \%$ agar medium plus the indicated sugars. Seed were stratified at $4{ }^{\circ} \mathrm{C}$ for 3 days and grown under $120-130 \mu \mathrm{E} \mathrm{m}^{-2} \mathrm{~s}^{-1}$ light under long day (16 h light) conditions with a mixture of fluorescent and incandescent lamps. All plant growth analyses were performed with at least three different biological replicates.

\section{CONSTRUCTION OF PROMOTER-REPORTER TRANSGENIC PLANTS AND IMAGING}

The promoter sequences of $S n R K 1.1$ (At3g01090) and SnRK1.2 (At3g29160) were analyzed using tools available from the web site Plant Cis-Acting Regulatory Element (P.L.A.C.E.) (Higo et al., 1999). We focused on using the native, intergenic regions of $S n R K 1.1$ and $S n R K 1.2$ genes, which is approximately 0.8 and $4.3 \mathrm{kB}$ of the $S n R K 1.1$ and $S n R K 1.25^{\prime}$ upstream sequences (i.e., promoters, respectively). These were amplified from CS60000 genomic DNA by PCR using the primers indicated in Supplemental Table 1, and were cloned via the Gateway system into plasmid pBGWFS7 (Karimi et al., 2002) containing an $e G F P:$ idA gene fusion. GUS constructs were transformed into Arabidopsis using Agrobacterium transformation (Bechtold et al., 1993). Homozygous lines were obtained through BASTA resistance screening. GUS staining of 3-10-day old grown on $0.5 \times$ MS agar plates + indicated sugars or of plant tissues from soil grown plants has been described (Styer et al., 2004), and staining was observed using an Olympus SZX16 and Zeiss Axiophot microscope. At least three biological replicates of different developmental stage and sugar-treated seedlings were performed.

\section{QUANTITATIVE PCR}

Conditions have been previously described (Donahue et al., 2010). Briefly, RNA was purified using a Qiagen RNeasy kit with DNase treatment, from 6 week-old soil-grown plants (roots, leaves, cauline leaves, flowers, siliques). Silique RNAs were initially extracted with phenol/chloroform and precipitated with LiCl before RNeasy purification. RNA was also extracted from seedlings grown on $0.5 \times$ MS agar plates for 5 days or 10 days. cDNA was synthesized from RNA using a Bio-Rad iScript cDNA synthesis kit. Reactions containing cDNA, Sybr Green MasterMix (Applied Biosystems) and primers, were performed in triplicate $\left(61^{\circ} \mathrm{C}\right.$ annealing temperature $)$ and monitored with Applied Biosystems 7300 Real-Time PCR instrumentation outfitted with SDS software version 1.4. Primers were designed to detect the longest SnRK1.1 cDNA (Accession no. AY093170), all known cDNAs encoding SnRK1.1, SnRK1.2 cDNA, and PEX4 cDNA (At5g25760) (Supplemental Table 1). For all experiments, at least two biological replicates were performed.

\section{FLOWERING TIME AND LEAF SIZE ASSAYS}

WT and mutant plants were grown as described previously under long-day ( $16 \mathrm{~h}$ days) conditions. Careful attention was given to growing plants side-by-side or in the same pot for comparison. Plants were examined at the point of inflorescence emergence and leaves were counted. Three biological replicates were analyzed. For leaf measurements the length and width of the largest leaf on 
each $27 \mathrm{~d}$ soil-grown plant was measured $(N=100-120$ plants per genotype).

\section{PROTEIN BLOT ANALYSES}

Conditions have been previously reported (Burnette et al., 2003). Briefly, tissues were ground in liquid nitrogen, homogenized, and resuspended with a pestle in SDS-bromophenol blue loading dye, boiled, and the supernatant was loaded onto a polyacrylamide gel for separation. Equal amounts of protein were loaded onto gels. SDS-PAGE was followed by western blotting with a 1:20,000 dilution of rabbit anti-GFP antibody (Invitrogen Molecular Probes, Eugene, OR) or an anti-SnRK1.1 antibody. This antibody was produced by CoCalico, Inc. by injecting rabbits with a SnRK1.1-V5 recombinant protein (Burnette et al., 2003) purified by ion-metal affinity (IMAC) and size exclusion chromatographies (Supplemental Figure 2). Sera from rabbits was purified by affinity-blot purification (as described in Harlow and Lane, 1988) and then tested for specificity with protein blots containing recombinant SnRK1.1, or extracts from SnRK1.1THA and previously characterized SnRK1.1 RNAi knock-down lines (Baena-Gonzalez et al., 2007) (Supplemental Figure 2). This anti-SnRK1.1 antibody does not cross-react with SnRK1.2GFP. A secondary goat, anti-rabbit horseradish peroxidase antibody (Bio-Rad Laboratories, Hercules, CA) was used at a 1:2500 dilution. Immunoreactive bands were detected using an ECL Plus Western Blotting Detection System (Amersham, Buckinghamshire, UK). Ponceau S staining of blots was performed to ensure that equal amounts of protein within extracts were analyzed.

\section{SnRK1 ACTIVITY ASSAYS}

The immunoprecipitation reactions were carried out as described (Ercetin et al., 2008) with the following modifications: leaves from mature $60 \mathrm{~d}$ plants were ground in liquid nitrogen and resuspended in extraction buffer $(50 \mathrm{mM}$ Tris-Cl, $\mathrm{pH} 7.5,150 \mathrm{mM}$ $\mathrm{NaCl}, 1 \mathrm{mM}$ EDTA, $10 \mathrm{mM} \mathrm{MgCl}_{2}, 0.1 \%$ Triton X-100, $10 \%$ glycerol) containing protease inhibitor (Sigma 9599), $10.5 \mu \mathrm{M}$ MG132 (proteasome inhibitor), and $10 \mathrm{mM}$ DTT. After centrifugation at $13.2 \mathrm{rpm}$ for $15 \mathrm{~min}$ the supernatant was incubated with either anti-GFP agarose or anti-HA antibody bound to protein A sepharose beads. Extracts and beads were rocked for $3 \mathrm{~h}$ at $4^{\circ} \mathrm{C}$, then washed $4 \times$ with Buffer $A$ and $2 \times$ with 50 mM HEPES, $\mathrm{pH} 7$, $1 \mathrm{mM}$ DTT. After washing, these beads were incubated in SnRK1 activity assays as described in Ananieva et al. (2008). Briefly, beads were incubated in kinase buffer $(50 \mathrm{mM}$ HEPES and $1 \mathrm{mM}$ DTT, $\mathrm{pH}$ 7.0), with SPS substrate peptide and $\gamma-{ }^{32} \mathrm{P}$-ATP, unlabeled ATP, and $\mathrm{MgCl}_{2}$. Samples were incubated for $30 \mathrm{~min}$ at $30^{\circ} \mathrm{C}$, and spotted onto P81 paper and washed in $125 \mathrm{mM}$ phosphoric acid as described. A reaction control with no SPS peptide to correct for autophosphorylation, and a no protein extract control were included. Activity is expressed as pmoles of phosphate incorporated into peptide per reaction. The assay was performed using two biologically independent extracts and three replicates of each extract. An initial time course with immunoprecipitated proteins indicated that product accumulation was linear over $45 \mathrm{~min}$ in these assay conditions (Supplemental Figure 2). Two biological replicates were analyzed.

\section{GFP LOCALIZATION AND IMAGING}

The coding regions of SnRK1.1, SnRK1.1T, and SnRK1.2 were amplified from plasmids or $7 \mathrm{~d}$ seedlings using the primers indicated in Supplemental Table 1, and were recombined into vector pK7FWG2 (Karimi et al., 2002) containing the 35S Cauliflower Mosaic virus promoter and a C-terminal GFP gene. Transformation of Arabidopsis was as described (Bechtold et al., 1993). Mature leaves of homozygous Arabidopsis lines were used for GFP fluorescence detection using a Zeiss LSM 510 laser-scanning microscope (Carl Zeiss) with an inverted Axio Observer Z1 base. GFP excitation was done using a 488-nm argon laser and fluorescence detected using 505- to $550-\mathrm{nm}$ band-pass emission filter. Slides were examined with $\times 40$ C-Apochromat water immersion lens. Nicotiana benthamiana plants were agroinfiltrated as previously described (Kapila et al., 1997). A set of mCherry tagged organelle markers were used for co-localization experiments (Nelson et al., 2007). Leaf sections were imaged $48 \mathrm{~h}$ post infiltration using the confocal microscope described above. mCherry was imaged using excitation with a 543-nm HeNe laser and 560-nm band-pass emission filter. At least three biological replicates were analyzed for each.

\section{RESULTS}

DEVELOPMENTAL REGULATION OF SnRK1.1 AND SnRK1.2 EXPRESSION

To explore the role SnRK1 may play in plant growth and development, we examined the spatial and temporal regulation of expression of the SnRK1.1 and SnRK1.2 genes. Promoter:gene reporter transgenic plants were constructed using 0.8 and $4.3 \mathrm{kB}$ of the SnRK1.1 and SnRK1.2 promoters, respectively. Multiple lines of SnRK1.1p:GUS and SnRK1.2p:GUS seedlings were identified, and homozygous lines were isolated and studied to verify reproducibility of GUS staining patterns. When SnRK1.1p:GUS and SnRK1.2p:GUS seedlings are grown on MS agar without an added carbon source, a striking difference in GUS expression is noted (Figures 1A-D). The SnRK1.1 promoter drives expression throughout the seedling, with highest expression in leaf primordia and vascular tissue (Figure 1A), and the seedling root tip (Figure 1C). In contrast, expression of the SnRK1.2 promoter is restricted to only a few cells at this stage including hydathodes, leaf primordial, and in portions of the cotyledon vascular tissue (Figures 1B,D). At 10 days both promoters drive strong expression in developing leaves (Figures 1E,F), with the SnRK1.2 promoter restricted to the base of the leaf, the vascular tissue, and the hydathodes (Figure 1F). Soil-grown plants were analyzed and indicate that $S n R K 1.1$ continues to be broadly expressed in the shoot (Figure 1G), whereas, activity of the SnRK1.2 promoter is restricted to the base of newly developing leaves and hydathodes (Figure 1H). In roots, expression of SnRK1.1 is abundant in vascular tissue and developing lateral root primordia with no added carbon source (Figures 1J,K, 2A,B), and in soil (Figure 1K). SnRK1.2 is not abundantly expressed in roots until $10 \mathrm{~d}$, at which time its expression is similar to that of SnRK1.1 (Figures 2A,B). SnRK1.1 is expressed in developing embryos within siliques, but SnRK1.2 is not (Figures 1L-O). We conclude that SnRK1.1 is more abundant and broadly expressed in plant tissues, whereas SnRK1.2 expression is more spatially restricted. 

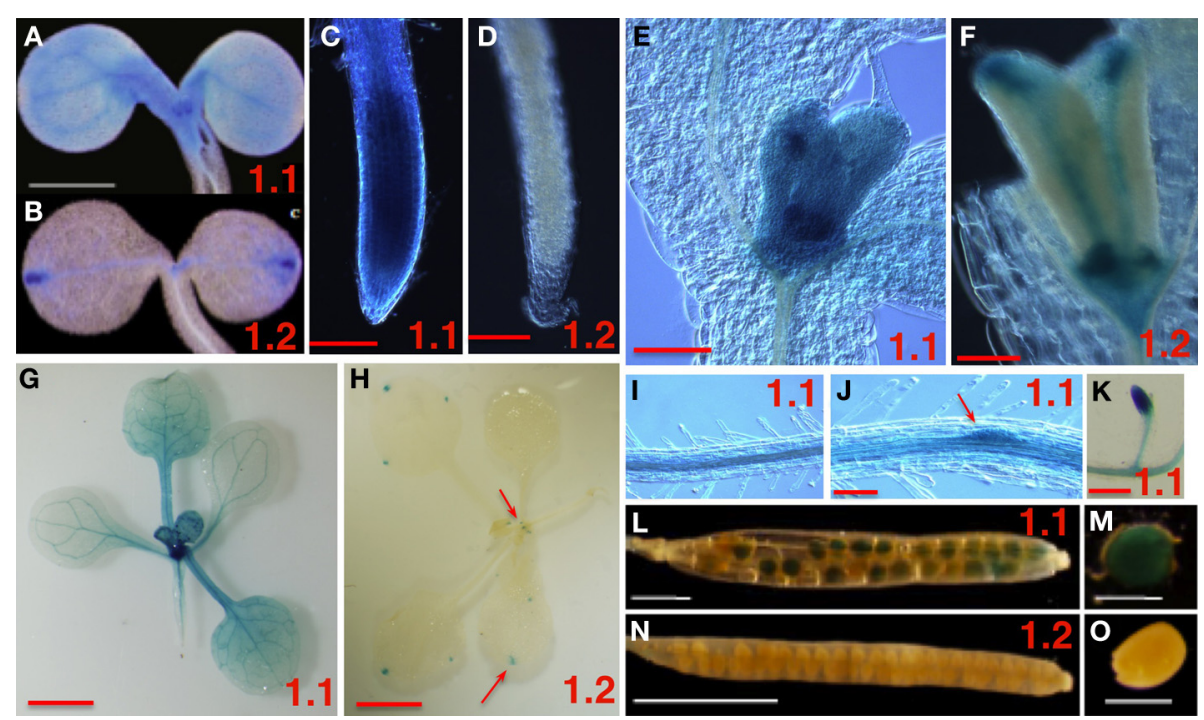

FIGURE 1 | Spatial Expression Patterns of SnRK1.1 and SnRK1.2 Genes. The promoters from SnRK1.1 (1.1 shown in $\mathbf{A}, \mathbf{C}, \mathbf{E}, \mathbf{G}, \mathbf{I}-\mathbf{M}$ ) or SnRK1.2 (1.2 shown in $\mathbf{B}, \mathbf{D}, \mathbf{F}, \mathbf{H}, \mathbf{N}, \mathbf{O})$ were used to drive GUS expression in transgenic plants. $(\mathbf{A}-\mathbf{F}, \mathbf{I}, \mathbf{J})$ are from plants grown on $\mathrm{MS}$ agar with no added sugar, and $(\mathbf{G}, \mathbf{H}, \mathbf{L}-\mathbf{O})$ are from soil-grown plants. $(\mathbf{A}, \mathbf{B}) \mathbf{5 d}$ cotyledons, bar $=1 \mathrm{~mm}$. (C,D) $5 \mathrm{~d}$ roots, bar $=100 \mu \mathrm{M}$. (E,F) $10 \mathrm{~d}$ leaf primordia, bar $=100 \mu \mathrm{M}$. (G,H,K) $15 \mathrm{~d}$ plants and roots, bar $=2 \mathrm{~mm}$. (I,J) $10 \mathrm{~d}$ roots, bar $=100 \mu \mathrm{M}$; $(\mathbf{L}, \mathbf{M})$ siliques, $(\mathbf{N}, \mathbf{O})$ developing seed, bar $=500 \mu \mathrm{M}$. The red arrows indicate small areas which are positive for staining.

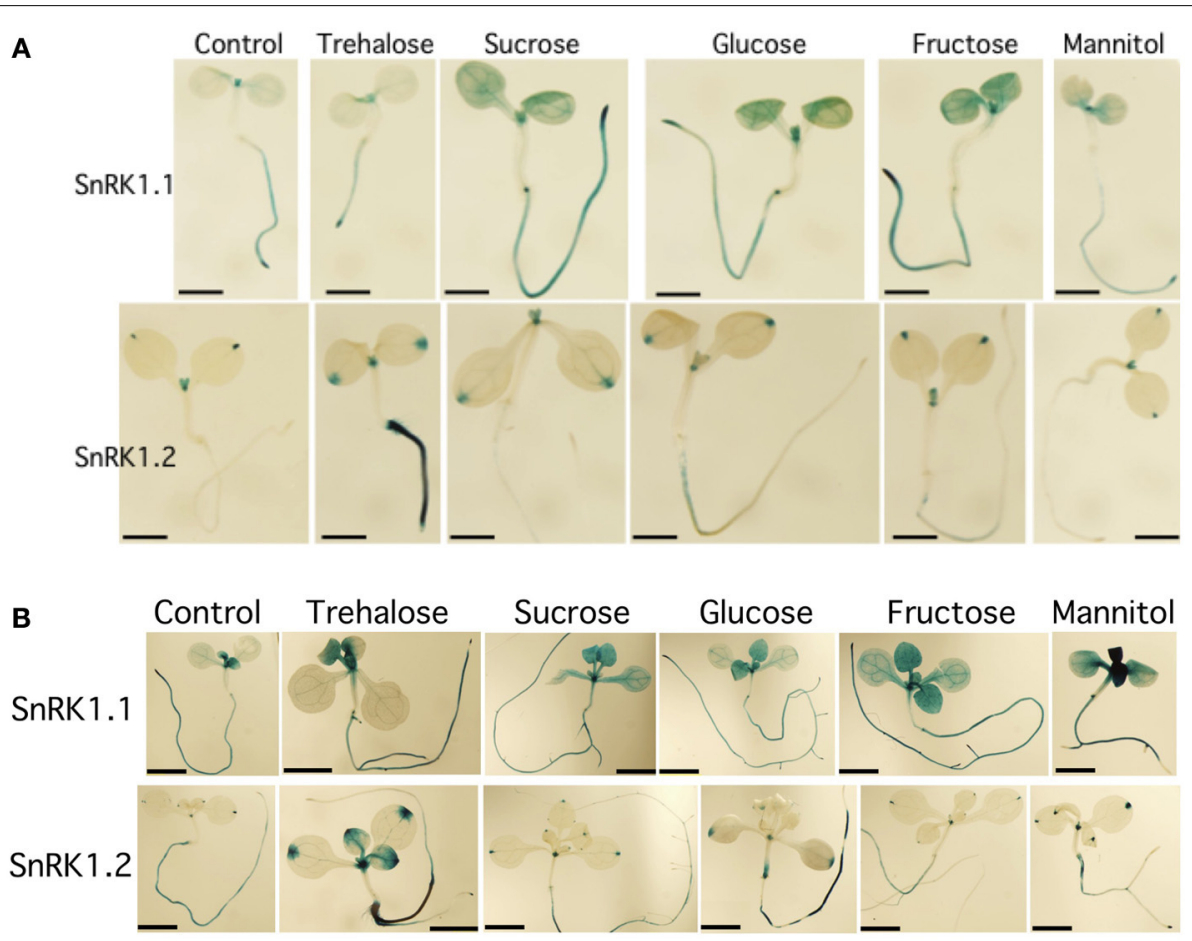

FIGURE 2 | Effects of sugars on the expression of SnRK1.1 and SnRK1.2. (A) $5 \mathrm{~d}$ seedlings grown with 1\% trehalose, 3\% sucrose, 1.5\% glucose, 1.5\% fructose or $3 \%$ mannitol. Bar $=1 \mathrm{~mm}$. (B) $10 \mathrm{~d}$ seedlings grown with the same sugars as in (A). Bar $=2 \mathrm{mM}$.

\section{REGULATION OF SnRK1.1 AND SnRK1.2 EXPRESSION BY SUGARS}

Because of the role of SnRK1 in carbon sensing and allocation in plants (Baena-Gonzalez et al., 2007; Jossier et al., 2009; Cho et al., 2012), we investigated whether different carbon sources would alter the patterning of SnRK1 expression as measured in SnRK1.1p:GUS and SnRK1.2p:GUS plants. In addition, we used quantitative RT-PCR (Q-PCR) to determine whether the abundance of SnRK1.1 and SnRK1.2 transcripts in wildtype plants were 
increased by sugar treatment. Seedlings grown for 5 and $10 \mathrm{~d}$ in the presence of trehalose, sucrose, glucose, fructose, or mannitol, did have small alterations in expression of SnRK1.1 in the shoot, but these changes may be indicative of changes in the developmental state (see GUS staining in cotyledons and new leaves in Figures 2A,B top panels). When a shorter-term treatment of sugar $(24 \mathrm{~h})$ is given to $9 \mathrm{~d}$ seedlings and SnRK1.1 expression is measured by Q-PCR, we find that $\operatorname{SnRK1.1}$ expression is slightly, but significantly, decreased by treatment with fructose, glucose, sucrose, but not trehalose or mannitol (Figure 3). In contrast, trehalose increases $S n R K 1.2$ expression, as seen by changes in

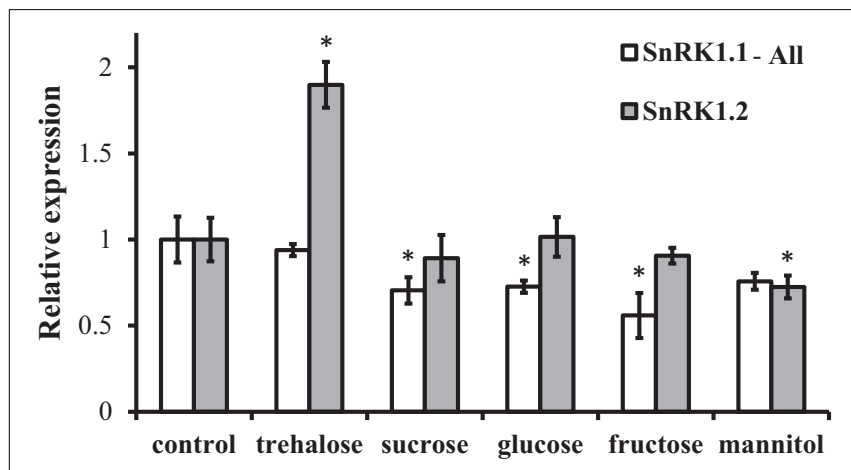

FIGURE 3 | RNA levels of SnRK1.1 and SnRK1.2 in sugar-treated seedlings. Wildtype seedlings were grown for 9 days and then treated with no sugar (control), $1 \%$ trehalose, $1.5 \%$ sucrose, $1.5 \%$ fructose, or $3 \%$ mannitol. SnRK1.1 and SnRK1.2 expression was measured with real-time PCR and was normalized to SnRK1.1 control and SnRK1.2 control levels, respectively, which are set to 1. SnRK1.1-All primers amplify all SnRK1.1 cDNAs, including those that encode SnRK1.1 and SnRK1.1T proteins. Means of triplicate reactions $\pm \mathrm{SD}$ are presented. ${ }^{*} p$-value $\leq 0.05$ when compared to the control.
GUS staining of SnRK1.2p:GUS seedlings grown for 5 or $10 \mathrm{~d}$ in trehalose (Figures 2A,B). Specifically, trehalose induced expression of SnRK1.2 in $5 \mathrm{~d}$ seedling roots that extended to the top of the root tip (Figure 2A, bottom panel). Trehalose also elevated $S n R K 1.2$ expression in $10 \mathrm{~d}$ seedling roots, hydathodes, vascular tissue, and the base of the leaf (Figure 2B, bottom panel). This increase in SnRK1.2 expression is also noted when a shorter-term treatment of sugar $(24 \mathrm{~h})$ is given to $9 \mathrm{~d}$ seedlings (Figure 3 ). In this short-term sugar treatment, mannitol also decreased SnRK1.2 expression to small degree (Figure 3). We conclude that trehalose increases $S n R K 1.2$ expression, while mannitol decreases $S n R K 1.2$ expression. Regulation of $S n R K 1.1$ differs as other sugars such as sucrose, glucose, and fructose result in a small decline in SnRK1.1 expression in seedlings.

\section{SnRK1.1 AND SnRK1.2 cDNAs AND SnRK1 PROTEIN ISOFORMS}

After noticing significant differences in a group of so-called fulllength $S_{n R K 1.1}$ cDNAs, we compared the structures of different SnRK1.1 and SnRK1.2 cDNAs (Figure 4A). For this analysis we focused solely on verified full-length cDNAs. Both SnRK1 genes are predicted to undergo differential splicing of a non-coding

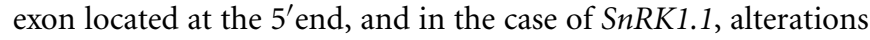
in splicing can result in two SnRK1.1 protein isoforms differing in a 23 amino acid extension at the N-terminus (Figures 4A,B). We have named the shorter truncated protein SnRK1.1T, while we call the longer protein SnRK1.1 (Figure 4B). Comparison of SnRK1.1, SnRK1.1T, and SnRK1.2 predicted proteins shows that the 23 amino acid extension is unique to SnRK1.1, while all three proteins contain highly similar sequences and protein domains (Figure 4B, Supplemental Figure 1). It is important to note that most investigations on $\operatorname{SnRK1.1}$ focus on the SnRK1.1T isoform, including the complementation of the yeast $s n f 1$ mutant (Alderson et al., 1991), and re-programming of Arabidopsis

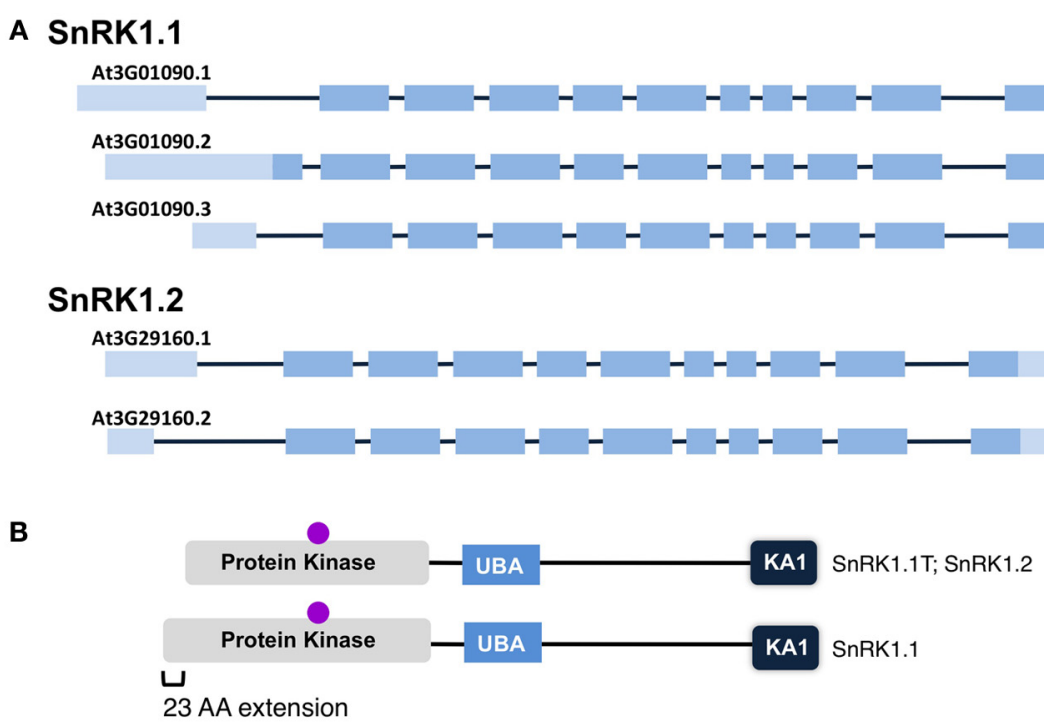

FIGURE 4 | Maps of SnRK1 Isoforms and Domain Structures. (A) The reported intron-exon maps of SnRK1 genes. Dark boxes denote exons while light boxes denote $5^{\prime}$ and $3^{\prime}$ UTRs. (B) SnRK1 kinases contain 3 domains: Protein Kinase, Ubiquitin Associated (UBA), and Kinase Associated 1 (KA1). The location of the active site residues are indicated by a purple circle. 
growth and ABA responses by overexpression (Baena-Gonzalez et al., 2007; Jossier et al., 2009).

We first used quantitative PCR to confirm the developmental expression patterns noted with promoter-GUS constructs, and to confirm that cDNAs capable of encoding the SnRK1.1 and SnRK1.1T proteins were expressed in Arabidopsis. For this work

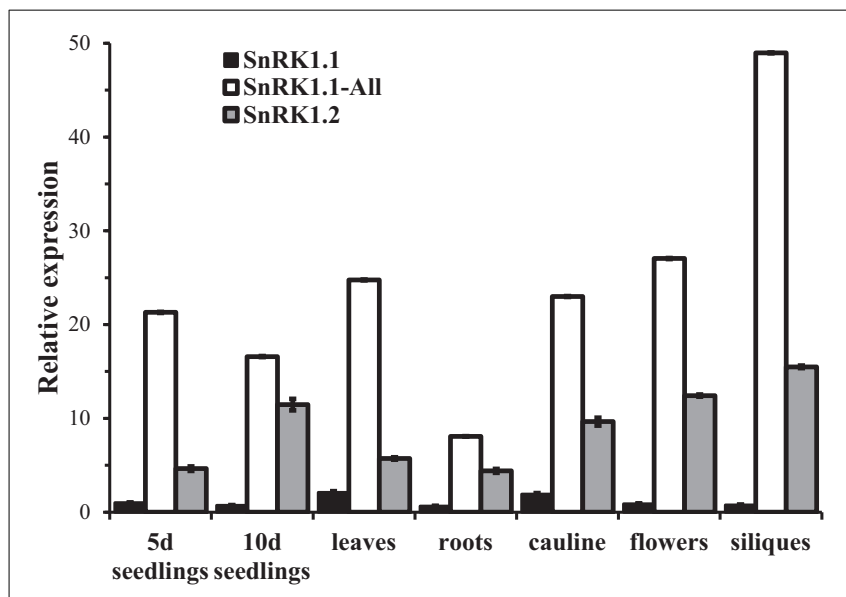

FIGURE 5 | Relative expression of SnRK1 genes as determined by real-time PCR. Primers specific for amplifying the longer cDNA clone that encodes the SnRK1.1 protein (SnRK1.1), all SnRK1.1 cDNAs (SnRK1.1-All), and $\mathrm{SnRK} 1.2$ were used to measure expression in $5 \mathrm{~d}$ and $10 \mathrm{~d}$ seedlings (seedlings), and tissues from 6 week-old soil-grown plants: rosette leaves, roots, cauline leaves, flowers, and green siliques. SnRK1 expression was compared to PEX4 to generate relative expression levels. Means of triplicate reactions \pm SD are presented. we designed oligonucleotide primers to amplify the unique $5^{\prime}$ end of the longer $S n R K 1.1$ cDNA that can encode the extra 23 amino acids (SnRK1.1), as well as the set of primers we previously used to amplify all SnRK1.1 cDNAs (called SnRK1.1-All), plus a set to amplify $S n R K 1.2$. We found very little expression of the longer SnRK1.1 isoform in all tissues examined (Figure 5). In contrast, our SnRK1.1-All primers detected expression at fairly high levels in seedlings, leaves, roots, cauline leaves, flowers, and siliques (Figure 5), indicating that the majority of SnRK1.1 expressed in these tissues has the ability to encode the SnRK1.1T protein isoform. SnRK1.2 was also expressed in these tissues, but at a lower level as compared to $S n R K 1.1$ (Figure 5).

\section{OVEREXPRESSION OF DIFFERENT SnRK1 PROTEINS IN PLANTA}

To determine whether the three SnRK1 protein isoforms (SnRK1.1, SnRK1.1T, and SnRK1.2) are each capable of altering plant growth and development, we compared the impact of overexpression of each. We used the 35S CaMV promoter to drive SnRK1-green fluorescent protein (GFP) gene fusions, and identified two independent lines with ectopic expression of SnRK1.1, SnRK1.1T, and SnRK1.2. Transgenic plants were characterized with respect to transgene expression using real time PCR. Figure 6 shows that our SnRK1.1-GFP, SnRK1.1TGFP, and SnRK1.2-GFP plants accumulate transgenic RNA (Figures 6A-C, respectively). In addition, we examined total SnRK1.1 and SnRK1.2 RNAs in these lines to confirm overexpression (Figures 6D-G). This analysis also showed that overexpression of the transgene was greater in some lines as compared to others, and could vary during development. For example, SnRK1.1a contains higher expression of SnRK1.1 than SnRK1.1b in seedlings, but not leaves (Figures 6A,B,D,E).
A
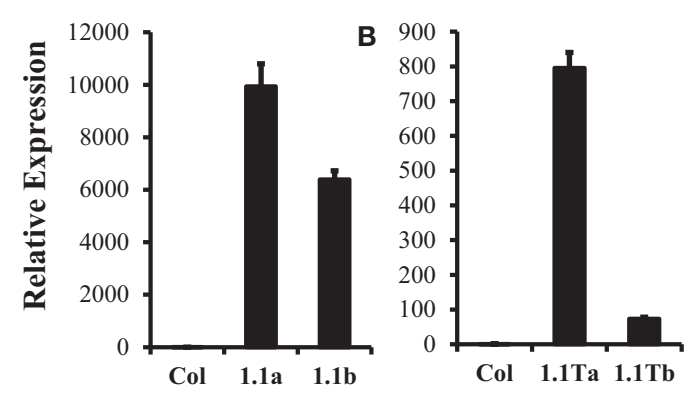

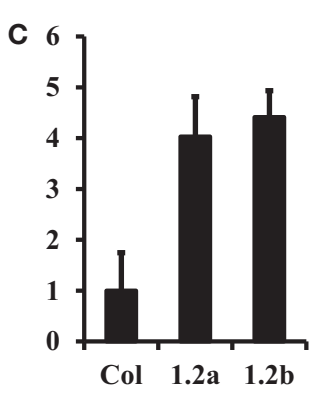

E
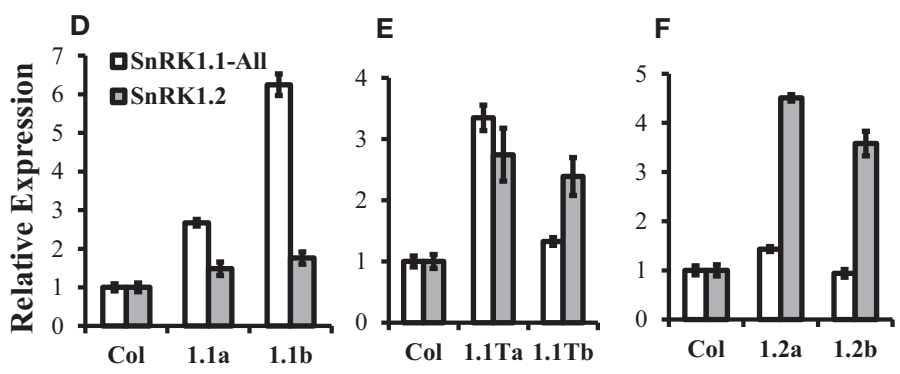

FIGURE 6 | Expression of Transgenes in SnRK1 transgenic plants. (A-C) Relative levels of GFP expression in $10 \mathrm{~d}$ seedlings of wild type (Col), SnRK1.1-GFP, SnRK1.1T-GFP, and SnRK1.2-GFP. (D-G)

Relative levels of $S n R K 1.1$ and $S n R K 1.2$ expression in the indicated lines with RNA extracted from (D-F) rosette leaves of $60 \mathrm{~d}$ soil-grown plants, and (G) $10 \mathrm{~d}$ seedlings. Means of triplicate reactions $\pm S D$ are presented. Two or more biological replicates were performed for each. 
Interestingly, SnRK1.2 levels were increased in SnRK1.1T-GFP plants (Figure 6E). (Figures 6F,G) indicate that SnRK1.2-GFP overexpression seedlings have a 2.4- and 2.9-fold increase in SnRK1.2 RNA (Figure 6F), while leaves from these same plants indicate a 4.5- and 3.6-fold increase in SnRK1.2 expression (Figure 6G).

We also wanted to verify that our SnRK1 transgenic plants accumulated active SnRK1 protein. Since removal of other contaminating kinases present in plant extracts is key to examining SnRK1 activity, we used immunoprecipitation of tagged SnRK1 proteins from plant extracts, followed by SnRK1 activity assays for this verification. Previously characterized Arabidopsis SnRK1 overexpressors constructed in the Landsberg erecta (Ler) ecotype (herein called SnRK1.1T-HA plants) were utilized as a positive control, and wildtype Arabidopsis Col and Ler plants were used as negative controls. Crude protein extracts from leaves were incubated with either anti-GFP agarose or anti-HA antibodies coupled to Protein A sepharose beads, and bound, washed proteins were incubated in SnRK1 activity assays with the SPS substrate peptide and $\gamma$ - $\mathrm{P}^{32}$-ATP as described previously (Ananieva et al., 2008) (Figure 7A). In addition, bound and washed proteins were analyzed by western blotting with anti-SnRK1.1, anti-GFP or anti-HA antibodies to examine the immunoprecipitated proteins (Figure 7B). The anti-SnRK1 antibody is described in detail in the Materials and Methods. As expected, we found that wildtype Col and Ler extracts had minimal (i.e., background) levels of SnRK1 activity (measured by the pmoles of $\mathrm{P}^{32}$ added to the SPS substrate peptide) (Figure 7A). In contrast, immunoprecipitated proteins from SnRK1.1-GFP, SnRK1.1T-GFP, and SnRK1.1THA plants indicated that all transgenic SnRK1.1 proteins are catalytically active (Figure 7A). However, immunoprecipitated

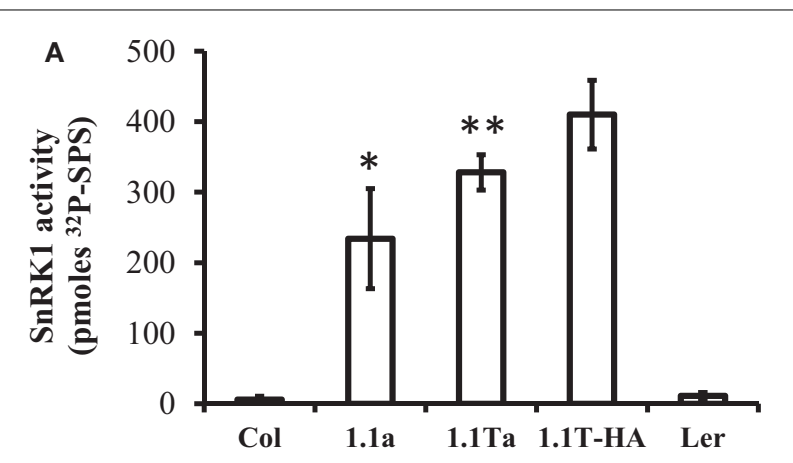

B

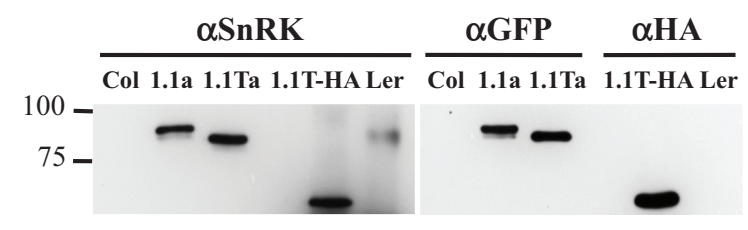

FIGURE 7 | SnRK1 activity in Transgenic Plants. (A) SnRK1 proeins were immunoprecipitated as described in the Materials and Methods, and kinase assays were performed. Means of 3 triplicate reactions \pm SD are presented. ${ }^{* *} p$-value $\leq 0.01$, and ${ }^{*}$ indicates a $p$-value less than 0.05 as compared to the 1.1T-HA sample. (B) An equal amount of sample was detected by western analysis with anti-SnRK, anti-GFP, or anti-HA antibody.
SnRK1.1-GFP has 57\%, and SnRK1.1T-GFP has $80 \%$, respectively, of the activity of SnRK1.1T-HA, suggesting that both GFP-tagged proteins have a reduction in their SnRK1 activity as compared to the HA-tagged protein. Western blotting with an anti-SnRK1 antibody verifies that proteins of the correct size were present in the immunoprecipitation, that anti-GFP and antiSnRK1 antibodies detect similarly sized proteins, and that the SnRK1.1T protein is slightly decreased in size from the larger, SnRK1.1 protein (Figure 7B). It is important to note that we tried several times to immunoprecipitate SnRK1.2-GFP from transgenic extracts, but could not obtain enough protein to do reliable activity assays. We could detect a faint band corresponding to SnRK1.2-GFP in both crude plant extracts and immunoprecipitations, so we conclude that SnRK1.2-GFP protein is being synthesized, however, the levels of this protein are very low, and preclude measuring enzyme activity.

\section{OVEREXPRESSION OF SnRK1.2 DECREASES TIME TO FLOWERING}

Previously characterized Arabidopsis SnRK1 overexpressers (SnRK1.1T-HA plants constructed in the Ler background) (Baena-Gonzalez et al., 2007), and SnRK1.1-HA plants constructed in the Col background (Tsai and Gazzarrini, 2012a), flower late, due to delayed developmental transitions throughout the life of the plant (Tsai and Gazzarrini, 2012b). This suggests that the 23 amino acid N-terminal extension missing in the SnRK1.1T protein does not impact function. Whether or not overexpression of SnRK1.2 has a similar effect on delaying developmental transitions has not been reported. To address these issues, we compared our SnRK1.1-GFP, SnRK1.1T-GFP, and SnRK1.2-GFP plants to the previously characterized SnRK1.1THA plants (see Supplemental Figure 3). We grew each transgenic line and matched wildtypes in different growth rooms and chambers set to standard long-day conditions in three separate experiments, and measured the days to flowering (Figure 8). As shown previously by others, SnRK1.1T-HA plants are significantly delayed in their flowering, by $3.3 \mathrm{~d}$ (Figure 8A). Both SnRK1.1-GFP lines also consistently flower late by app. $1 \mathrm{~d}$, and this difference is statistically significant for the SnRK1.1b line (Figure 8A). The SnRK1.1T-GFP lines also flower late by 1.9 and $1.2 \mathrm{~d}$, respectively (Figure 8A). Unexpectedly, both SnRK1.2-GFP lines flower early with a 3.6 and $4.5 \mathrm{~d}$ difference in days to flowering, respectively (Figure 8A). These same trends in flowering time were also seen when the number of leaves to flowering were measured, although in some cases the differences were not statistically significant (Figure 8B). We conclude that overexpression of SnRK1.2 has the opposite impact on flowering time as compared to that of SnRK1.1 overexpression. We also conclude that the reduction in SnRK1 activity caused by the GFP tag and the extra 23 aa present in SnRK1.1-GFP do not alter the ability of these proteins to induce late flowering when overexpressed, however, these changes may decrease the degree to which flowering time is delayed.

In addition to flowering early, we also noticed that SnRK1.2GFP plants tend to have flatter leaves (Figure 8C), that are $\sim 120 \%$ longer and $108 \%$ wider as compared to wildtype leaves (Supplemental Figure 4). Thus, overall, the appearance of SnRK1.2-GFP plants is altered when compared to wildtype or 


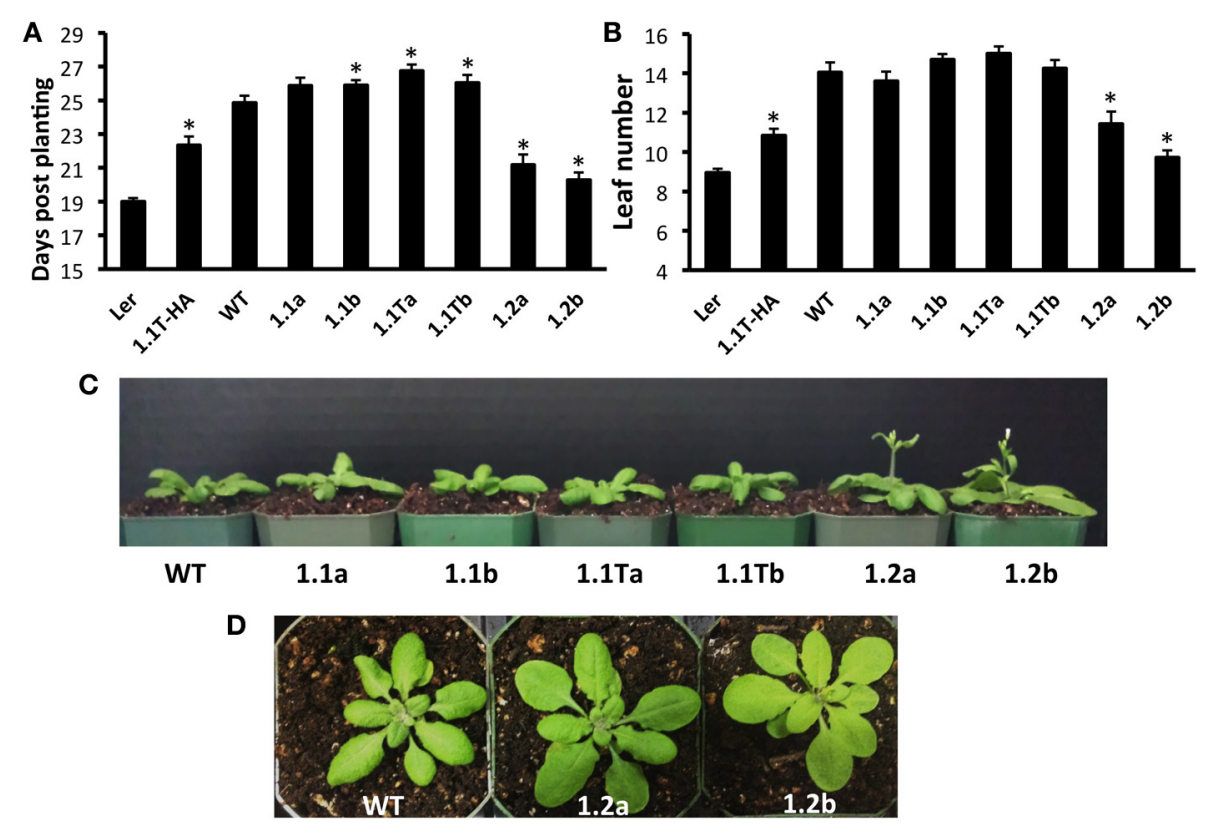

FIGURE 8 | Flowering Time Alterations in SnRK1 Transgenic Plants. (A) Days to flowering and (B) Number of leaves at flowering of soil-grown Arabidopsis wild type and SnRK1 overexpressing lines. (C) $21 \mathrm{~d}$ plants (D) 23 d plants. $N=30$ plants for each genotype. Means \pm SE are presented. ${ }^{*} p$-value $\leq 0.05$ when compared to the wildtype control. This experiment was replicated three times. the other SnRK1.1-GFP lines (Supplemental Figure 4). It is interesting to note that in our hands, the SnRK1.1T-HA plants are developmentally delayed until flowering, and then after flowering vegetative biomass increases, which is a potentially valuable trait (Supplemental Figure 4). To compare the growth and development of all SnRK1 transgenic lines, we examined growth throughout development, using rosette diameter as one indicator of organism size (Figure 9). This analysis shows that SnRK1.1THA plants have a smaller rosette size through $28 \mathrm{~d}$, and by $35 \mathrm{~d}$ their rosette diameter is larger (Figure 9). SnRK1.1-GFP and SnRK1.1T-GFP lines have a similar trend up to $28 \mathrm{~d}$, with both lines of each type showing smaller rosette diameter (Figure 9). In contrast, both SnRK1.2-GFP lines have a larger rosette diameter until $28 \mathrm{~d}$ (Figure 9). After $28 \mathrm{~d}$, data on rosette width starts to vary within each set of lines (Figure 9). Even though we tried to tightly control these experiments and repeated them with 3 biological replicates ( $N=100-120$ plants of each type), we have been unable to obtain consistent results on organism size of SnRK1-GFP plants during late stages of development. This is in stark contrast to the SnRK1.1T-HA plants that show very consistent increases in organismal size post-flowering (Figure 9, Supplemental Figure 4).

\section{SUBCELLULAR LOCALIZATION OF SnRK1.1, SnRK1.1T AND SnRK1.2}

To investigate the subcellular location of SnRK1.1, SnRK1.1T, and SnRK1.2, we first expressed each construct in N. benthamiana leaves in transient expression assays. To ensure the patterns observed were not due to overexpression and off-target accumulation, we used confocal microscopy to image cells at 12 , 24, 48, and $72 \mathrm{~h}$ after infiltration (data not shown). We found each SnRK1-GFP isoform was expressed and accumulated stably as measured by protein blotting (Figure 10, Top). $48 \mathrm{~h}$ after agro-infiltration, SnRK1.1-GFP localizes to the cytoplasm and nucleus, however the most striking signal is from large puncta that do not co-localize with chloroplasts (Figure 10A). To further investigate these puncta we transiently expressed SnRK1.1GFP with mCherry organelle markers in N. benthamiana. The SnRK1.1-GFP puncta do not co-localize with Golgi, peroxisomes, or mitochondria (Supplemental Figures 5A-C). We found that SnRK1.1T-GFP localizes to the cytoplasm and nucleus as does SnRK1.2-GFP (Figures 10B,C, respectively). While performing localization experiments, it was observed that SnRK1.1T-GFP plants form very small puncta in addition to the prior nuclear and cytoplasmic signal. The small puncta first appear in cells near the cut margin of the leaf, and increased over time after cutting and preparing samples for microscopy (Figure 10E). Similar smaller puncta were observed in SnRK1.1-GFP cells, however they took on the order of 30 more minutes to appear (Figure 10D). No large accumulation of puncta were observed in SnRK1.2-GFP cells, although there were areas in these cells close to the plasma membrane with a spotty pattern that appear to be distinct from the smaller puncta seen in SnRK1.1T-GFP and SnRK1.1GFP samples (Figure 10F). To determine the identity of these small puncta, co-localization experiments were performed with mCherry organelle markers. Small puncta from SnRK1-1T-GFP cells do not co-localize with Golgi, peroxisomal, or mitochondrial markers (Supplemental Figures 5D-F).

This pattern was confirmed in Arabidopsis with confocal imaging of homozygous progeny of two different overexpressing lines of SnRK1.1-GFP and SnRK1.1T-GFP plants. We found prominent large puncta in the SnRK1.1a line (Supplemental Figure 6A), and cytoplasmic localization in both 

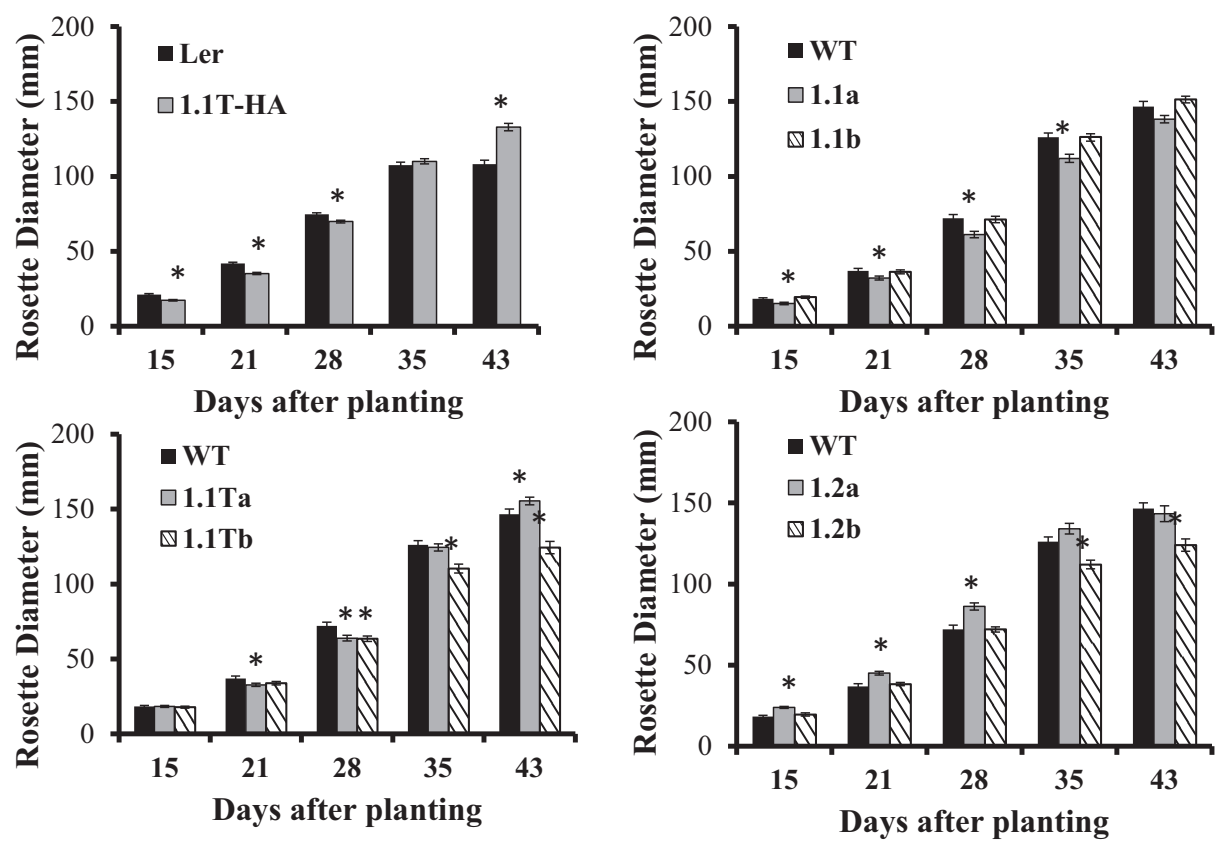

FIGURE 9 | Rosette Diameter Measurements. Rosette diameter of soil-grown plants grown under long day conditions for the indicated number of days. Means \pm SE are presented. * $p$-value $\leq 0.05$ when compared to the wildtype control.

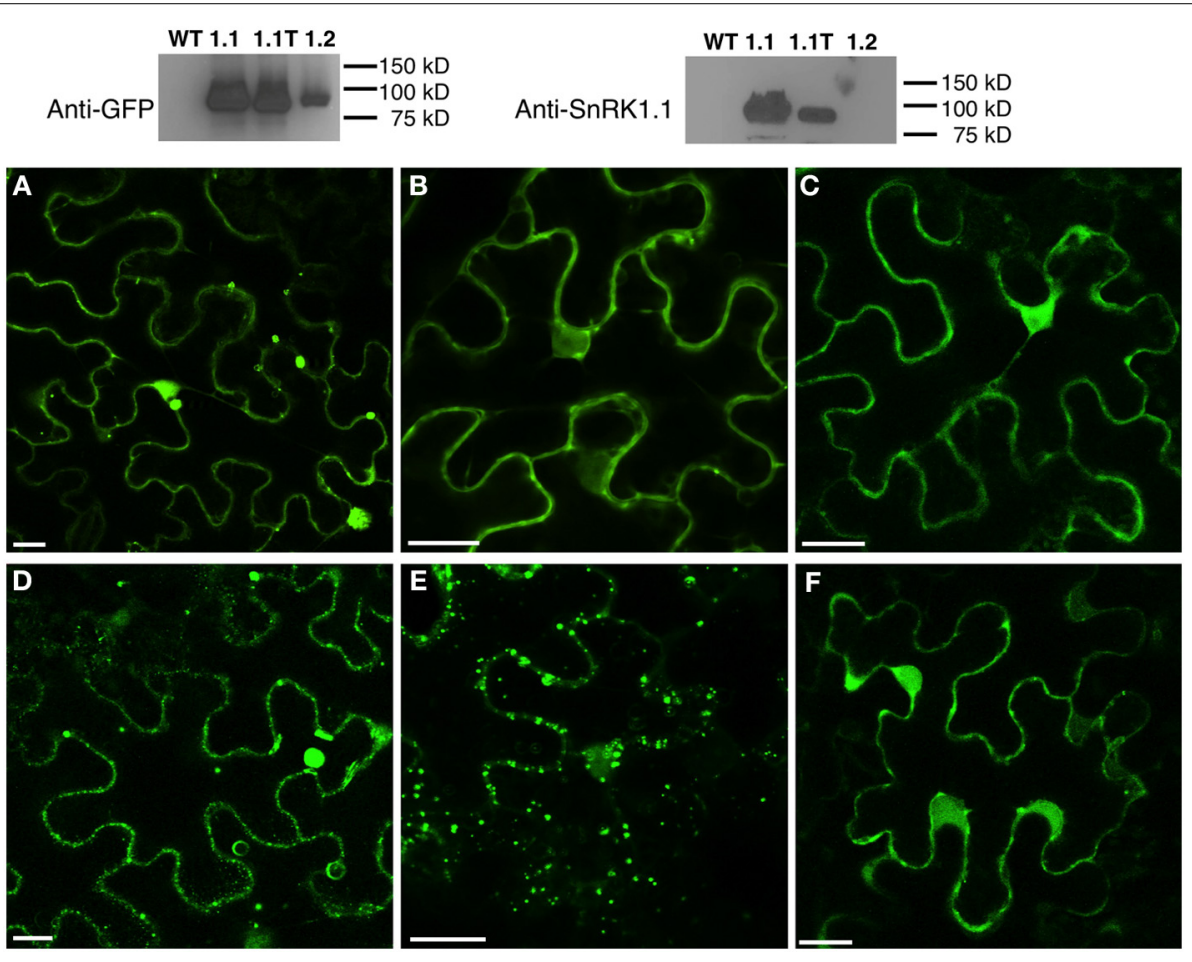

FIGURE 10 | Subcellular Location of SnRK1.1-, SnRK1.1T-, and SnRK1.2-GFP Proteins. Upper: Leaves of $N$. benthamiana transiently expressing SnRK1.1-GFP, SnRK1.1T-GFP, and SnRK1.2-GFP were analyzed by protein blotting with the indicated antiserum. Lower: Single optical sections of $N$. benthamiana transiently expressing SnRK1.1-GFP (A,D). SnRK1.1T-GFP (B,E), and SnRK1.2-GFP (C,F). A small section of mature leaves were removed and epidermal cells imaged using confocal microscopy at $0 \mathrm{~min}$ (A-C) and $30 \mathrm{~min}$ (D-F). Scale Bar $=20 \mu \mathrm{m}$. 
lines (Supplemental Figures 6A,B). SnRK1.1a is the line with more abundant expression (Figure 6). In SnRK1.1T-GFP plants we found that GFP fluorescence was cytoplasmic. Interestingly, small puncta began to appear in SnRK1.1Ta cells $60 \mathrm{~min}$ after mechanical wounding (i.e., cutting of the leaf margin) (Supplemental Figure 5E). We did not see the consistent nuclear signal observed in N. benthamiana for either Arabidopsis SnRK1.1 construct (compare Figure 10 and Supplemental Figure 5). We could not detect reliable GFP fluorescence in any of our SnRK1.2:GFP lines, thus we conclude that the SnRK1.2GFP protein is most likely below the level of detection using our confocal imaging conditions.

From both the N. benthamiana and Arabidopsis work we conclude that the SnRK1.1T isoform can localize to both the nucleus and cytoplasm, as well as being contained in small puncta of unknown origin. These puncta are stimulated by mechanical wounding and/or the stress of preparing samples, and may indicate that the SnRK1.1T isoform is dynamic and moves within the cell. The extra 23 amino acids on the N-terminus of SnRK1.1GFP is associated with the unique appearance of larger puncta that also are not part of a known organelle. Lastly, the SnRK1.2 isoform has a similar nuclear and cytoplasmic location, but does not accumulate in small puncta after wounding.

\section{DISCUSSION}

The SnRK1 protein is a major regulator of plant growth and development, and when overexpressed it can re-program metabolism (Baena-Gonzalez et al., 2007; Jossier et al., 2009) and delay developmental transitions (Tsai and Gazzarrini, 2012a). Since most previous work has focused on the SnRK1.1 gene, we sought to understand how SnRK1.1 and SnRK1.2 are regulated, and whether ectopic expression of each has the same or different consequences on plant growth and development. Our work provides key insights into spatial patterns of SnRK1.1 and SnRK1.2 expression, and also in the ability of SnRK1.2 to impact plant growth when overexpressed.

We used promoter:GUS transgenic plants and quantitative PCR to show that SnRK1.2 is much less abundantly expressed as compared to the SnRK1.1 gene (Figures 1, 2). In addition, although $S n R K 1.1$ is broadly expressed in both shoot and root tissues, SnRK1.2 expression is restricted to the hydathodes, cells at the base of leaf primordia, and some portions of vascular tissues. SnRK1.2 is also expressed in roots throughout development past $5 \mathrm{~d}$ (Figures 1,2). Given the previous biochemical and genetic studies by others showing that the SnRK1.2 is a protein kinase implicated in energy regulation (Baena-Gonzalez et al., 2007), we speculate that SnRK1.2 acts within a limited number of cells in the plant. We note here that this pattern of expression is similar to that from some genes involved in nutrient transport/sensing (Barker et al., 2000; Pilot et al., 2004). Our finding of restricted SnRK1.2 expression agrees with previous observations that SnRK1.1 is responsible for the major part of SnRK1 activity in Arabidopsis suspension cells (Jossier et al., 2009).

We also found that SnRK1.2 expression is elevated by trehalose (Figures 2, 3). Careful work by others has shown that trehalose mediates its effects via elevation of trehalose 6 phosphate (T6P) levels within plant cells (Schluepmann et al., 2004), where T6P is thought to communicate sugar or stress status (Schluepmann et al., 2003; O'Hara et al., 2013; Lastdrager et al., 2014). Previous microarray experiments have shown that $\operatorname{SnRK1.2}$ expression is elevated by trehalose, which suggested that SnRK1.2 participates in the signaling pathway to sense sugar or stress (Schluepmann et al., 2004). Our results bring spatial information to the trehalose regulation of SnRK1.2, indicating that this induction occurs primarily in the root area closest to the root-shoot junction. We speculate that roots, a sink tissue, may have a special need for responding to trehalose and $\mathrm{T} 6 \mathrm{P}$, as trehalose stimulates accumulation of $\sim 5$-fold more starch in source tissues such as the cotyledons and a corresponding decrease in starch in root columella cells (Aghdasi et al., 2010). Interestingly T6P is a regulator of flowering time (Wahl et al., 2013) and can also act as an inhibitor of SnRK1.1 and SnRK1.2 enzyme activity (Zhang et al., 2009; Delatte et al., 2011). Although it seems counter intuitive for T6P to increase SnRK1.2 transcription and decrease SnRK1.2 activity, it should be noted that T6P inhibition of SnRK1 activity requires an intermediary factor (Zhang et al., 2009). Thus it is possible that T6P dependence on this intermediary factor allows T6P to elevate SnRK1.2 transcription in the root, while decreasing activity elsewhere.

One of the most compelling reasons to study SnRK1 function in plants is the alteration of growth and development conferred by overexpression of SnRK1 (McKibbin et al., 2006; Baena-Gonzalez et al., 2007; Tsai and Gazzarrini, 2012a) (Supplemental Figure 4), which could be a useful tool for engineering desirable traits (Coello et al., 2011). Specifically, others have shown that overexpression of SnRK1.1 delays developmental transitions, such as the vegetative to reproductive transition, which manifests as a delay in time to flowering (Tsai and Gazzarrini, 2012a). This delay effectively decreases the size of SnRK1.1 overexpressors early in development, as measured by a smaller rosette width (Figure 9). Similarly, we found that SnRK1.1-GFP and SnRK1.1T-GFP plants flowered late and had smaller rosettes up to $28 \mathrm{~d}$ (Figures 8, 9). In contrast, overexpression of SnRK1.2, which was accompanied by increased levels of SnRK1.2 RNA (Figure 6), resulted in early flowering (Figure 8), and larger rosettes and leaves prior to day 28 (Figure 9, Supplemental Figure 4). These data indicate that SnRK1.2, when overexpressed, has the opposite impact on flowering time as compared to SnRK1.1 overexpression. Previous biochemical studies have supported a similar enzyme activity for SnRK1.1 and SnRK1.2 proteins (Baena-Gonzalez et al., 2007; Jossier et al., 2009), and knockdown of SnRK1.1 and SnRK1.2 in Arabidopsis also support a redundant role for these two proteins. The mechanism for how overexpression of SnRK1.2 leads to a change in growth and shortens time to flowering is unknown at present. We speculate that the SnRK1.2 gene, which is normally restricted in expression (in hydathodes, at the base of leaf primordia, and in vascular tissues within both seedling shoots and roots), could impact changes in flowering time gene regulation. As we see very little SnRK1.2:GFP protein accumulation in our overexpression lines, we suggest that a small difference in SnRK1.2 expression may have significant effects due to elevated protein kinase activity and impact on signaling. This concept of slight changes in kinase activity triggering significant downstream effects by 
amplifying signals has been discussed previously (Chock et al., 1980).

Another possibility for the seemingly opposite impact of SnRK1.1 and SnRK1.2 overexpression, is that SnRK1.1 and SnRK1.2 physically interact with different protein partners in the cell, and such interactors could be key for driving different biological outcomes in SnRK1 overexpression plants. In support of this, a recent query of String proteome-wide binary protein-protein interactions (Szklarczyk et al., 2011) indicated that SnRK1.1 and SnRK1.2 have some novel interactors. These include two different senescence-associated proteins, along with FUS3 and FUS5 for SnRK1.1, and JAZ3, the TOE2 transcription factor, and Starch Excess 4 (SEX4) for SnRK1.2. It is interesting to note that sex4 mutants show a prolonged juvenile stage, and flower late (Matsoukas et al., 2013), thus this potentially novel SnRK1.2 interactor has a known connection to regulation of flowering time.

To further understand SnRK1.2 function, we performed subcellular localization studies using both a transient expression assay, and by examining transformed Arabidopsis. We found SnRK1.2 localized to both the nucleus and cytoplasm, which is the same location we and others have documented for SnRK1.1 (Lopez-Paz et al., 2009; Bitrian et al., 2011; Tsai and Gazzarrini, 2012a; Mohannath et al., 2014). An intriguing finding is that SnRK1.1T-GFP is stimulated by mechanical wounding to localize in small puncta (Figure 9, Supplemental Figure 6). These small puncta are not part of known organelles such as chloroplasts, mitochondria, peroxisomes, or the Golgi apparatus (Supplemental Figure 5). These puncta have been previously noted by others (Lopez-Paz et al., 2009; Bitrian et al., 2011; Tsai and Gazzarrini, 2012a), but our report is the first to connect these to a mechanical wounding stimulus. It is known that rice SnRK1.1T-GFP most likely moves between the nucleus and cytoplasm (Cho et al., 2012). We found SnRK1.1T in both the nucleus and cytoplasm in transient expression assays, but could not confirm the nuclear location in mature Arabidopsis leaves. Others reported SnRK1.1T-GFP in the chloroplast of stably transformed Arabidopsis (Fragoso et al., 2009). While we cannot rule out that some portion of SnRK1.1T-GFP localized to chloroplasts and it was below our limits of detection in our studies, our data strongly support the cytoplasm and small puncta as areas of the cell where SnRK1.1T-GFP accumulates to the highest degree. We speculate that the small puncta allow for movement of SnRK1.1T between compartments in response to stress. In addition, since we never observed SnRK1.2GFP in small puncta or formation in response to wounding, we hypothesize that movement within the cell is unique to SnRK1.1 isoforms.

We also addressed the existence of a longer SnRK1.1 cDNA that is predicted to encode a SnRK1.1 protein with 23 extra amino acids at the N-terminus. We found only minimal expression of this SnRK1.1 cDNA (Figure 5), and the SnRK1.1-GFP encoded by this cDNA had an additional subcellular location of accumulation within large puncta (Figure 9). SnRK1.1-GFP transiently expressed also localized to small puncta after wounding, although there was a delay in appearance of these puncta. Understanding what role, if any, this SnRK1.1 isoform plays in plants awaits purification of native SnRK1.1 isoforms and analysis via mass spectrometry.

In conclusion, the data reported here support a role for $\operatorname{SnRK1.2}$ as a spatially restricted $\operatorname{SnRK1} 1$ isoform that is capable of inducing early flowering when overexpressed. In addition, our results indicate an intriguing new possibility that certain stresses, such as mechanical wounding, induce movement or redistribution of SnRK1.1T protein in the cell.

\section{ACKNOWLEDGMENTS}

The authors acknowledge the NSF and NIFA for funding portions of this work (NSF MCB\#1051646 and NIFA 2013-67013-21356 to Glenda E. Gillaspy). The Virginia Agricultural Experiment Research Station is also acknowledged. We also thank Elena Baena-Gonzalez and Filip Rolland for making 35S-SnRK1.1T-HA plants available, and Elitsa Ananieva for construction of clones and helpful advice.

\section{SUPPLEMENTARY MATERIAL}

The Supplementary Material for this article can be found online at: http://www.frontiersin.org/journal/10.3389/fpls.2014.00324/ abstract

Supplemental Figure 1 | Amino acid alignment of SnRK1 proteins.

Predicted amino acid sequences of SnRK1.1, SnRK1.1T, and SnRK1.2 were aligned with ClustalW.

Supplemental Figure 2 | Anti-SnRK1 Antibody and Activity Assay. (A) Purified SnRK1.1-V5 recombinant protein was separated by SDS-PAGE.

(B,D) Immunoreactivity of anti-SnRK1.1 with native SnRK1.1. Plant extracts were made from wildtype Ler plants (WT), SnRK1.1.T-HA (1.1T-HA), and SnRK1.1 RNAi knock-down lines 10-1 and 10-2 (Baena-Gonzalez et al., 2007). Protein blots were probed with a 1:1500 dilution of purified anti-SnRK1.1 (B) or 1:2500 dilution of a commercial anti-HA antibody (D). (C) Linearity of SnRK1 activity assay conditions. Means \pm SE are presented.

Supplemental Figure 3 | Overexpression of SnRK1.1 in Ler. Transgenic plants overexpressing SnRK1.1T-HA (SnRK1-Ox) and matched control WT (Ler) were grown under LD conditions.

Supplemental Figure 4 | Appearance and leaf size measurements of SnRK1 transgenic plants. (A) $30 \mathrm{~d}$ wildtype and transgenic plants. (B) Leaf length and width measurements taken at $27 \mathrm{~d}$.

Supplemental Figure 5 | Colocalization of SnRK-GFP with organelle markers. Single optical sections of $N$. benthamiana transiently expressing SnRK1.1-GFP (A-C) and SnRK1.1T-GFP (D,E). SnRK-GFP was

co-expressed with a set of mCherry tagged organelle markers, golgi (A,D), peroxisome (B,E), and mitochondria (C,F). Epidermal cells of mature leaves were imaged using confocal microscopy. Bar $=20 \mu \mathrm{m}$.

Supplemental Figure 6 | Subcellular localization of SnRK1.1 and SnRK1.1T in Arabidopsis. Epidermal cells of mature leaves of soil grown Arabidopsis plants imaged with confocal microscopy. Two independent lines of SnRK1.1:GFP [1.1a (A) and 1.1b (B)] and SnRK1.1T:GFP [1.1Tb (C) and 1.1Ta (D)] were imaged immediately after cutting leaf sections. The two lines of SnRK1.2:GFP gave no detectable signal. Leaf sections were imaged again after 30 and $60 \mathrm{~min}$. The SnRK 1.1Ta contain small fluorescent puncta $\sim 60 \mathrm{~min}$ after wounding (E). Bar $=20 \mu \mathrm{m}$. 


\section{REFERENCES}

Aghdasi, M., Schluepmann, H., and Smeekens, S. (2010). Characterization of Arabidopsis seedlings growth and development under trehalose feeding. J. Cell Mol. Res. 1, 1-9.

Alderson, A., Sabelli, P. A., Dickinson, J. R., Cole, D., Richardson, M., Kreis, M., et al. (1991). Complementation of snf1, a mutation affecting global regulation of carbon metabolism in yeast, by a plant protein kinase cDNA. Proc. Natl. Acad. Sci. U.S.A. 88, 8602-8605. doi: 10.1073/pnas.88.19.8602

Ananieva, E. A., Gillaspy, G. E., Ely, A., Burnette, R. N., and Erickson, F. L. (2008). Interaction of the WD40 domain of a myoinositol polyphosphate 5phosphatase with SnRK1 links inositol, sugar, and stress signaling. Plant Physiol. 148, 1868-1882. doi: 10.1104/pp.108.130575

Baena-Gonzalez, E., Rolland, F., Thevelein, J. M., and Sheen, J. (2007). A central integrator of transcription networks in plant stress and energy signalling. Nature 448, 938-942. doi: 10.1038/nature06069

Barker, L., Kuhn, C., Weise, A., Schulz, A., Gebhardt, C., Hirner, B., et al. (2000). SUT2, a putative sucrose sensor in sieve elements. Plant Cell 12, 1153-1164. doi: 10.1105/tpc.12.7.1153

Bechtold, N., Ellis, J., and Pelletier, G. (1993). In planta Agrobacterium mediated gene transfer by infiltration of adult Arabidopsis thaliana plants. C. R. Acad. Sci. Ser. III Sci. Vie 316, 1194-1199.

Bitrian, M., Roodbarkelari, F., Horvath, M., and Koncz, C. (2011). BACrecombineering for studying plant gene regulation: developmental control and cellular localization of SnRK1 kinase subunits. Plant J. 65, 829-842. doi: 10.1111/j.1365-313X.2010.04462.x

Bradford, K. J., Downie, A. B., Gee, O. H., Alvarado, V., Yang, H., and Dahal, P. (2003). Abscisic acid and gibberellin differentially regulate expression of genes of the SNF1-related kinase complex in tomato seeds. Plant Physiol. 132, 1560-1576. doi: 10.1104/pp.102.019141

Burnette, R. N., Gunesekera, B. M., and Gillaspy, G. E. (2003). An Arabidopsis inositol 5-phosphatase gain-of-function alters abscisic acid signaling. Plant Physiol. 132, 1011-1019. doi: 10.1104/pp.019000

Cho, Y. H., Hong, J. W., Kim, E. C., and Yoo, S. D. (2012). Regulatory functions of SnRK1 in stress-responsive gene expression and in plant growth and development. Plant Physiol. 158, 1955-1964. doi: 10.1104/pp.111.189829

Chock, P. B., Rhee, S. G., and Stadtman, E. R. (1980). Interconvertible enzyme cascades in cellular regulation. Ann. Rev. Biochem. 49, 813-843. doi: 10.1146/annurev.bi.49.070180.004121

Coello, P., Hey, S. J., and Halford, N. G. (2011). The sucrose non-fermenting1-related (SnRK) family of protein kinases: potential for manipulation to improve stress tolerance and increase yield. J. Exp. Bot. 62, 883-893. doi: 10.1093/jxb/erq331

Confraria, A., Martinho, C., Elias, A., Rubio-Somoza, I., and Baena-Gonzalez, E. (2014). miRNAs mediate SnRK1-dependent energy signaling in Arabidopsis. Front. Plant Sci. 4:197. doi: 10.3389/fpls.2013.00197

Delatte, T. L., Sedijani, P., Kondou, Y., Matsui, M., De Jong, G. J., Somsen, G. W., et al. (2011). Growth arrest by trehalose-6-phosphate: an astonishing case of primary metabolite control over growth by way of the SnRK1 signaling pathway. Plant Physiol. 157, 160-174. doi: 10.1104/pp.111.180422

Donahue, J. L., Alford, S. R., Torabinejad, J., Kerwin, R. E., Nourbakhsh, A., Ray, W. K., et al. (2010). The Arabidopsis thaliana Myo-inositol 1-phosphate synthase1 gene is required for $M y o$-inositol synthesis and suppression of cell death. Plant Cell 22, 888-903. doi: 10.1105/tpc.109.071779

Ercetin, M. E., Ananieva, E. A., Safaee, N. M., Torabinejad, J., Robinson, J. Y., and Gillaspy, G. E. (2008). A phosphatidylinositol phosphate-specific myo-inositol polyphosphate 5-phosphatase required for seedling growth. Plant Mol. Biol. 67, 375-388. doi: 10.1007/s11103-008-9327-3

Fragoso, S., Espindola, L., Paez-Valencia, J., Gamboa, A., Camacho, Y., MartinezBarajas, E., et al. (2009). SnRK1 isoforms AKIN10 and AKIN11 are differentially regulated in Arabidopsis plants under phosphate starvation. Plant Physiol. 149, 1906-1916. doi: 10.1104/pp.108.133298

Gazzarrini, S., Tsuchiya, Y., Lumba, S., Okamoto, M., and McCourt, P. (2004). The transcription factor FUSCA3 controls developmental timing in Arabidopsis through the hormones gibberellin and abscisic acid. Dev. Cell 7, 373-385. doi: 10.1016/j.devcel.2004.06.017

Ghillebert, R., Swinnen, E., Wen, J., Vandesteene, L., Ramon, M., Norga, K., et al. (2011). The AMPK/SNF1/SnRK1 fuel gauge and energy regulator: structure, function and regulation. FEBS J. 278, 3978-3990. doi: 10.1111/j.17424658.2011.08315.x
Gissot, L., Polge, C., Jossier, M., Girin, T., Bouly, J. P., Kreis, M., et al. (2006). AKINbetagamma contributes to SnRK1 heterotrimeric complexes and interacts with two proteins implicated in plant pathogen resistance through its KIS/GBD sequence. Plant Physiol. 142, 931-944. doi: 10.1104/pp.106.087718

Halford, N. G., Hey, S., Jhurreea, D., Laurie, S., McKibbin, R. S., Paul, M., et al. (2003). Metabolic signalling and carbon partitioning: role of Snfl-related (SnRK1) protein kinase. J. Exp. Bot. 54, 467-475. doi: 10.1093/jxb/erg038

Halford, N. G., and Hey, S. J. (2009). Snf1-related protein kinases (SnRKs) act within an intricate network that links metabolic and stress signalling in plants. Biochem. J. 419, 247-259. doi: 10.1042/BJ20082408

Halford, N. G., and Paul, M. J. (2003). Carbon metabolite sensing and signalling. Plant Biotechnol. J. 1, 381-398. doi: 10.1046/j.1467-7652.2003.00046.x

Harlow, E., and Lane, D. (1988). Antibodies a Laboratory Manual. New York, NY: Cold Spring Harbor Laboratory.

Hey, S. J., Byrne, E., and Halford, N. G. (2010). The interface between metabolic and stress signalling. Ann. Bot. 105, 197-203. doi: 10.1093/aob/mcp285

Higo, K., Ugawa, Y., Iwamoto, M., and Korenaga, T. (1999). Plant cis-acting regulatory DNA elements (PLACE) database: 1999. Nucleic Acids Res. 27, 297-300. doi: 10.1093/nar/27.1.297

Jossier, M., Bouly, J. P., Meimoun, P., Arjmand, A., Lessard, P., Hawley, S., et al. (2009). SnRK1 (SNF1-related kinase 1) has a central role in sugar and ABA signalling in Arabidopsis thaliana. Plant J. 59, 316-328. doi: 10.1111/j.1365313X.2009.03871.x

Kapila, J., Derycke, R., Van Montagu, M., and Angenon, G. (1997). An Agrobacterium-mediated transient gene expression system for intact leaves. Plant Sci. 122, 101-108. doi: 10.1016/S0168-9452(96)04541-4

Karimi, M., Inze, D., and Depicker, A. (2002). GATEWAY vectors for Agrobacterium-mediated plant transformation. Trends Plant Sci. 7, 193-195. doi: 10.1016/S1360-1385(02)02251-3

Kleinow, T., Bhalerao, R., Breuer, F., Umeda, M., Salchert, K., and Koncz, C. (2000). Functional identification of an Arabidopsis snf4 ortholog by screening for heterologous multicopy suppressors of snf4 deficiency in yeast. Plant J. 23, 115-122. doi: 10.1046/j.1365-313x.2000.00809.x

Lastdrager, J., Hanson, J., and Smeekens, S. (2014). Sugar signals and the control of plant growth and development. J. Exp. Bot. 65, 799-807. doi: 10.1093/jxb/ert474

Lin, C. R., Lee, K. W., Chen, C. Y., Hong, Y. F., Chen, J. L., Lu, C. A., et al. (2014). SnRK1A-interacting negative regulators modulate the nutrient starvation signaling sensor SnRK1 in source-sink communication in cereal seedlings under abiotic stress. Plant Cell 26, 808-827. doi: 10.1105/tpc.113.121939

Lopez-Paz, C., Vilela, B., Riera, M., Pages, M., and Lumbreras, V. (2009). Maize AKINbetagamma dimerizes through the KIS/CBM domain and assembles into SnRK1 complexes. FEBS Lett. 583, 1887-1894. doi: 10.1016/j.febslet.2009.05.022

Lumbreras, V., Alba, M. M., Kleinow, T., Koncz, C., and Pages, M. (2001). Domain fusion between SNF1-related kinase subunits during plant evolution. EMBO Rep. 2, 55-60. doi: 10.1093/embo-reports/kve001

Matsoukas, I. G., Massiah, A. J., and Thomas, B. (2013). Starch metabolism and antiflorigenic signals modulate the juvenile-to-adult phase transition in Arabidopsis. Plant Cell Environ. 36, 1802-1811. doi: 10.1111/pce.12088

McKibbin, R. S., Muttucumaru, N., Paul, M. J., Powers, S. J., Burrell, M. M., Coates, S., et al. (2006). Production of high-starch, low-glucose potatoes through overexpression of the metabolic regulator SnRK1. Plant Biotechnol. J. 4, 409-418. doi: 10.1111/j.1467-7652.2006.00190.x

Mohannath, G., Jackel, J. N., Lee, Y. H., Buchmann, R. C., Wang, H., Patil, V., et al. (2014). A complex containing SNF1-related kinase (SnRK1) and adenosine kinase in Arabidopsis. PLoS ONE 9:e87592. doi: 10.1371/journal.pone. 0087592

Nelson, B. K., Cai, X., and Nebenfuhr, A. (2007). A multicolored set of in vivo organelle markers for co-localization studies in Arabidopsis and other plants. Plant J. 51, 1126-1136. doi: 10.1111/j.1365-313X.2007.03212.x

Ng, S., Giraud, E., Duncan, O., Law, S. R., Wang, Y., Xu, L., et al. (2013). Cyclin-dependent kinase E1 (CDKE1) provides a cellular switch in plants between growth and stress responses. J. Biol. Chem. 288, 3449-3459. doi: 10.1074/jbc.M112.416727

O'Hara, L. E., Paul, M. J., and Wingler, A. (2013). How do sugars regulate plant growth and development? New insight into the role of trehalose-6-phosphate. Mol. Plant 6, 261-274. doi: 10.1093/mp/sss120

Pien, S., Wyrzykowska, J., and Fleming, A. J. (2001). Novel marker genes for early leaf development indicate spatial regulation of carbohydrate metabolism 
within the apical meristem. Plant J. 25, 663-674. doi: 10.1046/j.1365313x.2001.01002.x

Pilot, G., Stransky, H., Bushey, D. F., Pratelli, R., Ludewig, U., Wingate, V. P., et al. (2004). Overexpression of GLUTAMINE DUMPER1 leads to hypersecretion of glutamine from Hydathodes of Arabidopsis leaves. Plant Cell 16, 1827-1840. doi: $10.1105 /$ tpc. 021642

Polge, C., Jossier, M., Crozet, P., Gissot, L., and Thomas, M. (2008). Beta-subunits of the SnRK1 complexes share a common ancestral function together with expression and function specificities; physical interaction with nitrate reductase specifically occurs via AKINbetal-subunit. Plant Physiol. 148, 1570-1582. doi: 10.1104/pp.108.123026

Ramon, M., Ruelens, P., Li, Y., Sheen, J., Geuten, K., and Rolland, F. (2013). The hybrid four-CBS-domain KINbetagamma subunit functions as the canonical gamma subunit of the plant energy sensor SnRK1. Plant J. Cell Mol. Biol. 75, 11-25. doi: $10.1111 /$ tpj. 12192

Rodrigues, A., Adamo, M., Crozet, P., Margalha, L., Confraria, A., Martinho, C., et al. (2013). ABI1 and PP2CA phosphatases are negative regulators of Snf1related protein kinase1 signaling in Arabidopsis. Plant Cell 25, 3871-3884. doi: 10.1105/tpc.113.114066

Rolland, F., Baena-Gonzalez, E., and Sheen, J. (2006). Sugar sensing and signaling in plants: conserved and novel mechanisms. Annu. Rev. Plant Biol. 57, 675-709. doi: 10.1146/annurev.arplant.57.032905.105441

Schluepmann, H., Pellny, T., Van Dijken, A., Smeekens, S., and Paul, M. (2003). Trehalose 6-phosphate is indispensable for carbohydrate utilization and growth in Arabidopsis thaliana. Proc. Natl. Acad. Sci. U.S.A. 100, 6849-6854. doi: 10.1073/pnas.1132018100

Schluepmann, H., Van Dijken, A., Aghdasi, M., Wobbes, B., Paul, M., and Smeekens, S. (2004). Trehalose mediated growth inhibition of Arabidopsis seedlings is due to trehalose-6-phosphate accumulation. Plant Physiol. 135, 879-890. doi: 10.1104/pp.104.039503

Singh, B., Cheek, H. D., and Haigler, C. H. (2009). A synthetic auxin (NAA) suppresses secondary wall cellulose synthesis and enhances elongation in cultured cotton fiber. Plant Cell Rep. 28, 1023-1032. doi: 10.1007/s00299-009-0714-2

Styer, J. C., Keddie, J., Spence, J., and Gillaspy, G. E. (2004). Genomic organization and regulation of the LeIMP-1 and LeIMP-2 genes encoding myo-inositol monophosphatase in tomato. Gene 326, 35-41. doi: 10.1016/j.gene.2003.09.048

Szklarczyk, D., Franceschini, A., Kuhn, M., Simonovic, M., Roth, A., Minguez, P., et al. (2011). The STRING database in 2011: functional interaction networks of proteins, globally integrated and scored. Nucleic Acids Res. 39, D561-D568. doi: 10.1093/nar/gkq973
Takano, M., Kajiya-Kanegae, H., Funatsuki, H., and Kikuchi, S. (1998). Rice has two distinct classes of protein kinase genes related to SNF1 of Saccharomyces cerevisiae, which are differently regulated in early seed development. Mol. Gen. Genet. 260, 388-394. doi: 10.1007/s004380050908

Thelander, M., Olsson, T., and Ronne, H. (2004). Snf1-related protein kinase 1 is needed for growth in a normal day-night light cycle. EMBO J. 23, 1900-1910. doi: 10.1038/sj.emboj.7600182

Tsai, A. Y., and Gazzarrini, S. (2012a). AKIN10 and FUSCA3 interact to control lateral organ development and phase transitions in Arabidopsis. Plant J. 69, 809-821. doi: 10.1111/j.1365-313X.2011.04832.x

Tsai, A. Y., and Gazzarrini, S. (2012b). Overlapping and distinct roles of AKIN10 and FUSCA3 in ABA and sugar signaling during seed germination. Plant Signal. Behav. 7, 1238-1242. doi: 10.4161/psb.21549

Tsai, A. Y., and Gazzarrini, S. (2014). Trehalose-6-phosphate and SnRK1 kinases in plant development and signaling: the emerging picture. Front. Plant Sci. 5:119. doi: $10.3389 /$ fpls.2014.00119

Wahl, V., Ponnu, J., Schlereth, A., Arrivault, S., Langenecker, T., Franke, A., et al. (2013). Regulation of flowering by trehalose-6-phosphate signaling in Arabidopsis thaliana. Science 339, 704-707. doi: 10.1126/science.1230406

Zhang, Y., Primavesi, L. F., Jhurreea, D., Andralojc, P. J., Mitchell, R. A., Powers, S. J., et al. (2009). Inhibition of SNF1-related protein kinasel activity and regulation of metabolic pathways by trehalose-6-phosphate. Plant Physiol. 149, 1860-1871. doi: 10.1104/pp.108.133934

Conflict of Interest Statement: The authors declare that the research was conducted in the absence of any commercial or financial relationships that could be construed as a potential conflict of interest.

Received: 31 January 2014; accepted: 21 June 2014; published online: 10 July 2014. Citation: Williams SP, Rangarajan P, Donahue JL, Hess JE and Gillaspy GE (2014) Regulation of Sucrose non-Fermenting Related Kinase 1 genes in Arabidopsis thaliana. Front. Plant Sci. 5:324. doi: 10.3389/fpls.2014.00324

This article was submitted to Plant Physiology, a section of the journal Frontiers in Plant Science.

Copyright (๑) 2014 Williams, Rangarajan, Donahue, Hess and Gillaspy. This is an open-access article distributed under the terms of the Creative Commons Attribution License (CC BY). The use, distribution or reproduction in other forums is permitted, provided the original author(s) or licensor are credited and that the original publication in this journal is cited, in accordance with accepted academic practice. No use, distribution or reproduction is permitted which does not comply with these terms. 